\title{
Calix[4]thiacrowns as Ditopic Hosts for Homo- and Heterobinuclear Accommodation: First Report of a 'Chopsticks-type' $\pi$-coordination
}

\author{
Jai Young Lee, ${ }^{t, \neq}$ Jongchul Kwon, ${ }^{\ddagger}$ Chul Soon Park, ${ }^{\ddagger}$ Ji-Eun Lee, ${ }^{\dagger}$ Wonbo Sim, ${ }^{*, \neq}$ \\ Jong Seung Kim, ${ }^{\S}$ Joobeom Seo, ${ }^{\dagger}$ II Yoon, ${ }^{\dagger}$ Jong Hwa Jung, ${ }^{\dagger}$ and Shim Sung \\ Lee $^{\star, \dagger}$
}

$\dagger$ Department of Chemistry (BK21) and Institute of Natural Science, Gyeongsang National University, Jinju 660-701, Korea,

$\$$ Department of Chemistry, Konyang University, Nonsan 320-711, Korea,

§Department of Chemistry, Dankook University, Seoul 140-714, Korea

sslee@gsnu.ac.kr (S.S.Lee),wsim@konyang.ac.kr(W.Sim)

Synthesis of $\mathbf{L}^{1}$. To a refluxed solution of $\mathrm{Cs}_{2} \mathrm{CO}_{3}(1.67 \mathrm{~g}, 5.10 \mathrm{mmol})$ in THF $(80 \mathrm{~mL})$ was added dropwise a solution of $3(1.00 \mathrm{~g}, 1.02 \mathrm{mmol})$ and 3,6-dioxa-1,8-octanedithiol (0.28 $\mathrm{g}, 1.53 \mathrm{mmol})$ in THF (100 $\mathrm{mL})$ for $3 \mathrm{~h}$ under nitrogen, and the reaction mixture was refluxed for an additional $48 \mathrm{~h}$. After cooling to room temperature, $10 \% \mathrm{HCl}(10 \mathrm{~mL})$ was added and the solvent was removed under reduced pressure. The reaction mixture was extracted with $\mathrm{CH}_{2} \mathrm{Cl}_{2}(3 \times 50 \mathrm{~mL})$, washed with water and then dried over anhydrous $\mathrm{MgSO}_{4}$. The crude product was chromatographed on silica gel using ethyl acetate and $n$-hexane (1:3) as eluent, and recrystallized from $\mathrm{CH}_{2} \mathrm{Cl}_{2} / n$-hexane $(1: 30, v / v)$ to give a white crystalline solid in $60 \%$ yield (0.50 g). Mp 171 172 ${ }^{\circ} \mathrm{C}$. IR (KBr pellet): 2891, 1445, 1299, 1247, 1217, 1147, 1093, 1000, 921, 765, 634, 509, $445 \mathrm{~cm}^{-1} .{ }^{1} \mathrm{H}$ NMR $\left(\mathrm{CDCl}_{3}\right): \delta 7.08 \sim 6.84(\mathrm{~m}, 12 \mathrm{H}, A r), 3.88(\mathrm{~s}, 8 \mathrm{H}$, $\left.\mathrm{ArCH}_{2} \mathrm{Ar}\right), 3.72\left(\mathrm{~s}, 4 \mathrm{H}, \mathrm{CH}_{2} \mathrm{OCH}_{2}\right), 3.66\left(\mathrm{t}, 4 \mathrm{H}, \mathrm{ArOCH}_{2} \mathrm{CH}_{2} \mathrm{~S}, J=6.3 \mathrm{~Hz}\right.$ ), $3.49(\mathrm{~s}, 8 \mathrm{H}$, $\mathrm{OCH}_{2} \mathrm{CH}_{2} \mathrm{O}$ ), 3.46 (t, $4 \mathrm{H}, \mathrm{OCH}_{2} \mathrm{CH}_{2} \mathrm{~S}, J=8.2 \mathrm{~Hz}$ ), 3.33 (t, $4 \mathrm{H}, \mathrm{ArOCH}_{2} \mathrm{CH}_{2} \mathrm{O}, \quad J=6.3 \mathrm{~Hz}$ ), 3.07 (t, $4 \mathrm{H}, \mathrm{ArOCH}_{2} \mathrm{CH}_{2} \mathrm{O}, J=6.3 \mathrm{~Hz}$ ), 2.58 (t, $4 \mathrm{H}, \mathrm{ArOCH}_{2} \mathrm{CH}_{2} \mathrm{~S}, J=6.3 \mathrm{~Hz}$ ), 1.94 (t, $8 \mathrm{H}$, $\left.\mathrm{SCH}_{2} \mathrm{CH}_{2} \mathrm{O}, J=8.2 \mathrm{~Hz}\right) .{ }^{13} \mathrm{C} \mathrm{NMR}\left(\mathrm{CDCl}_{3}\right): \delta 156.5122,155.9595,134.0823,133.9223$, 129.1003, 128.9039, 122.9764, 122.6927, 72.6399, 71.0399, 70.8871, 70.6253, 69.9125, 69.1925, 69.3488, 38.0859, 32.0347, 30.1874 ppm. MS (FAB, $m / z): 839.94\left(\mathrm{M}^{+}+\mathrm{Na}\right), 816.96$ $\left(\mathrm{M}^{+}\right)$. 


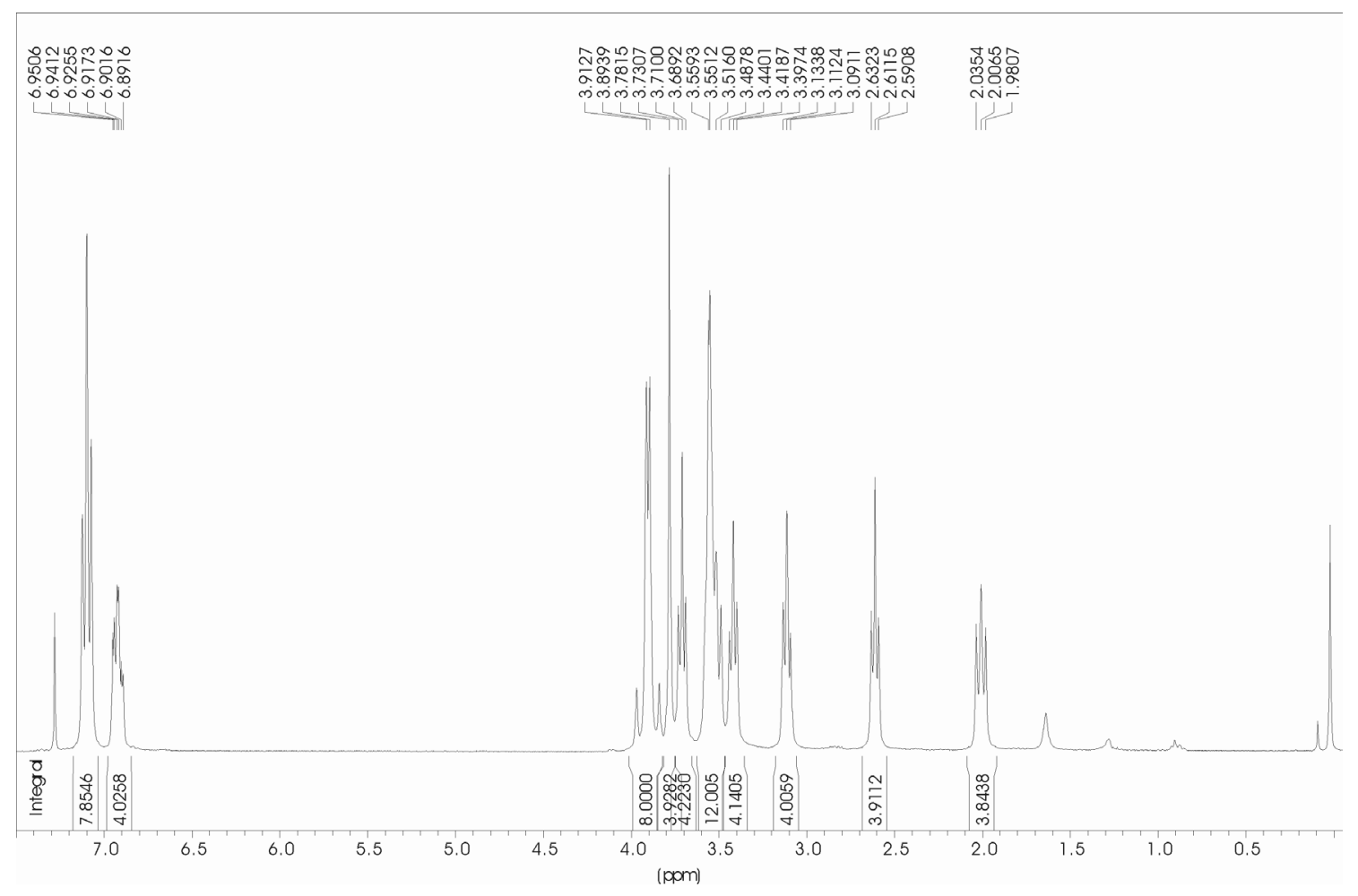

Figure S1. ${ }^{1} \mathrm{H}$ NMR spectrum of $\mathbf{L}^{\mathbf{1}}$.

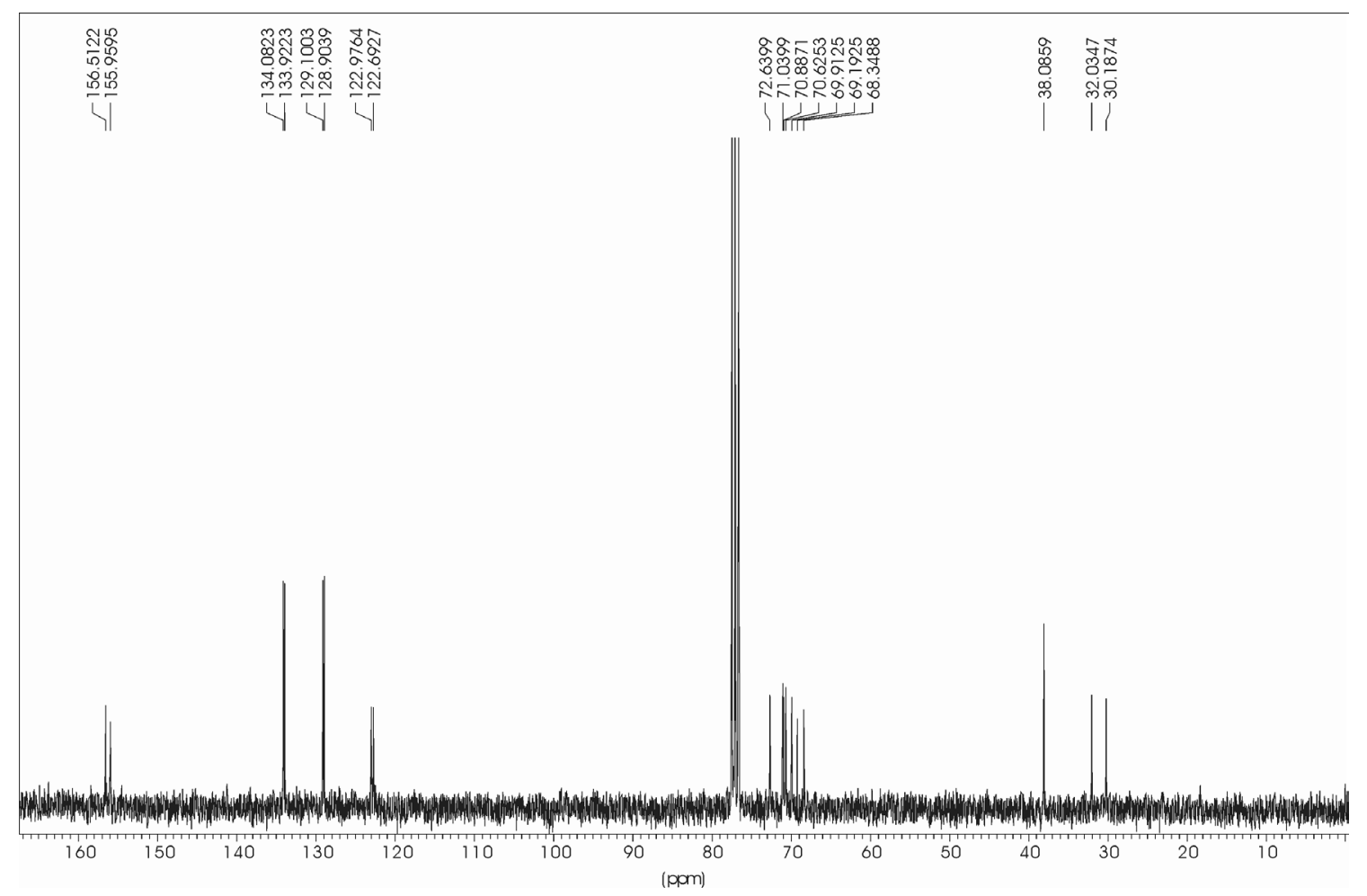

Figure S2. ${ }^{13} \mathrm{C}$ NMR spectrum of $\mathbf{L}^{\mathbf{1}}$. 
Synthesis of $\mathbf{L}^{2}$. To a refluxed solution of $\mathrm{Cs}_{2} \mathrm{CO}_{3}(2.69 \mathrm{~g}, 8.21 \mathrm{mmol})$ in THF (80 mL) was added dropwise a solution of $6(1.00 \mathrm{~g}, 0.82 \mathrm{mmol})$ and 3,6-dioxa-1,8-octanedithiol (0.38 $\mathrm{g}, 2.05 \mathrm{mmol})$ in THF $(100 \mathrm{~mL})$ for $3 \mathrm{~h}$ under nitrogen, and the reaction mixture was refluxed for an additional $48 \mathrm{~h}$. After cooling to room temperature, $10 \% \mathrm{HCl}(10 \mathrm{~mL})$ was added and the solvent was removed under reduced pressure. The reaction mixture was extracted with $\mathrm{CH}_{2} \mathrm{Cl}_{2}(3 \times 50 \mathrm{~mL})$, washed with water and then dried over anhydrous $\mathrm{MgSO}_{4}$. The crude product was chromatographed on silica gel using ethyl acetate and $n$-hexane (1:3) as eluent, and recrystallized from $\mathrm{CH}_{2} \mathrm{Cl}_{2} / n$-hexane $(1: 30, v / v)$ to give a white crystalline solid in $48 \%$ yield (0.35 g). Mp 256 $258{ }^{\circ} \mathrm{C}$. IR (KBr pellet): 2902, 1430, 1369, 1245, 1128, 1093, 1012, 919, 763, $632 \mathrm{~cm}^{-1} .{ }^{1} \mathrm{H}$ NMR $\left(\mathrm{CDCl}_{3}\right): \delta 7.05$ (d, $\left.8 \mathrm{H}, \mathrm{Ar}-\mathrm{H}\right), 6.90(\mathrm{t}, 4 \mathrm{H}, \mathrm{Ar}-\mathrm{H}), 3.88(\mathrm{~s}, 8 \mathrm{H}$, $\mathrm{ArCH}_{2} \mathrm{Ar}$ ), 3.74 (s, $8 \mathrm{H}, \mathrm{CH}_{2} \mathrm{OCH}_{2}$ ), 3.67(t, 8H, $\left.\mathrm{ArOCH}_{2} \mathrm{CH}_{2} \mathrm{~S}, J=6.2 \mathrm{~Hz}\right), 3.49$ (t, $8 \mathrm{H}$, $\mathrm{SCH}_{2} \mathrm{CH}_{2} \mathrm{O}, J=8.1 \mathrm{~Hz}$ ), 2.57 (t, $8 \mathrm{H}, \mathrm{ArOCH}_{2} \mathrm{CH}_{2} \mathrm{~S}, J=6.2 \mathrm{~Hz}$ ), 2.00 (t, $8 \mathrm{H}, \mathrm{SCH}_{2} \mathrm{CH}_{2} \mathrm{O}$, $J=8.1 \mathrm{~Hz}) .{ }^{13} \mathrm{C} \mathrm{NMR}\left(\mathrm{CDCl}_{3}\right): \delta 156.1558,133.8277,128.8530,122.8600,71.0399,70.8799$, 69.0252, 38.1222, 32.0856, 30.3547 ppm. MS (FAB, m/z): $915.93\left(\mathrm{M}^{+}+\mathrm{Na}\right), 892.97\left(\mathrm{M}^{+}\right)$.

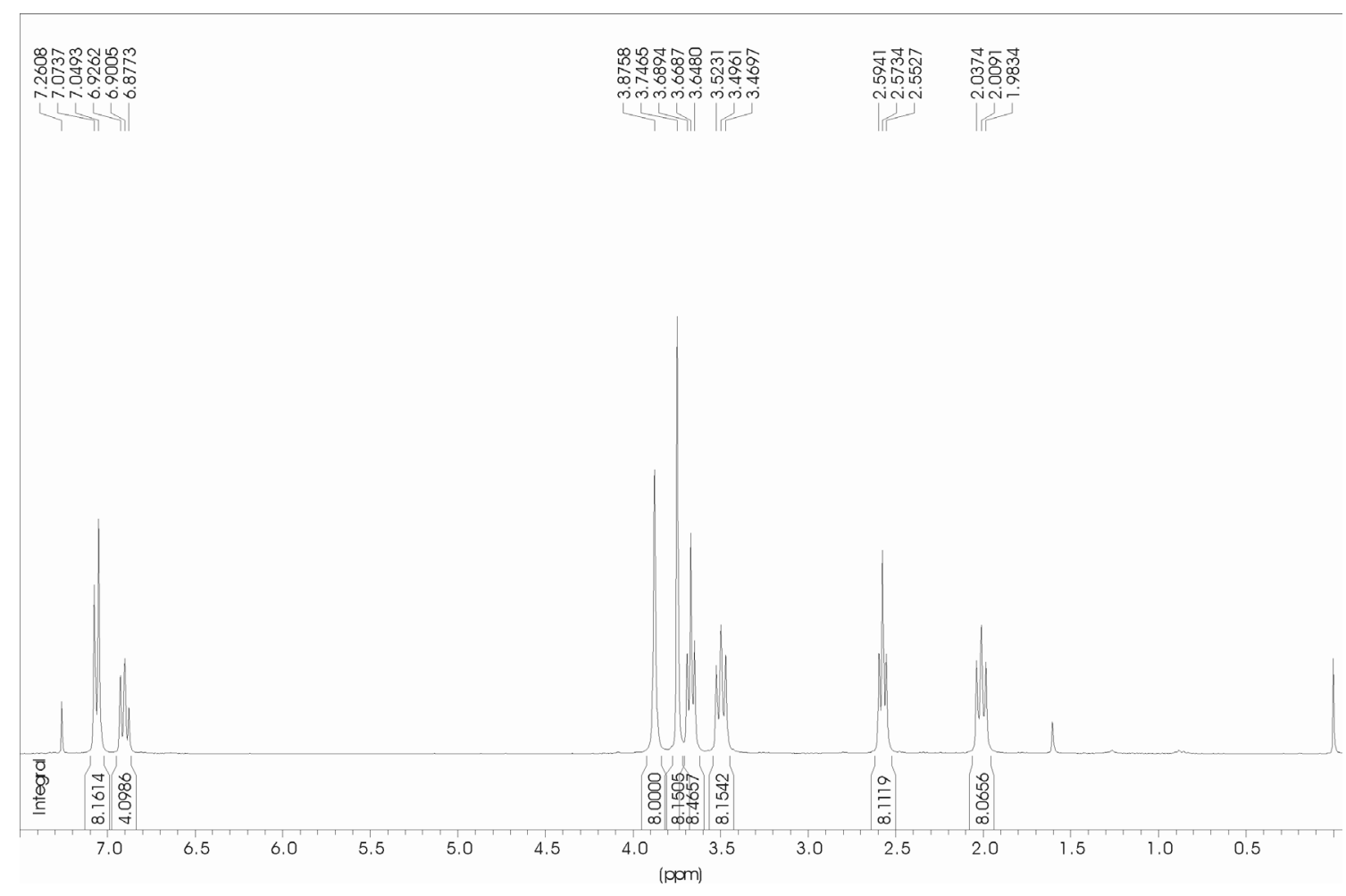

Figure S3. ${ }^{1} \mathrm{H}$ NMR spectrum of $\mathbf{L}^{2}$. 


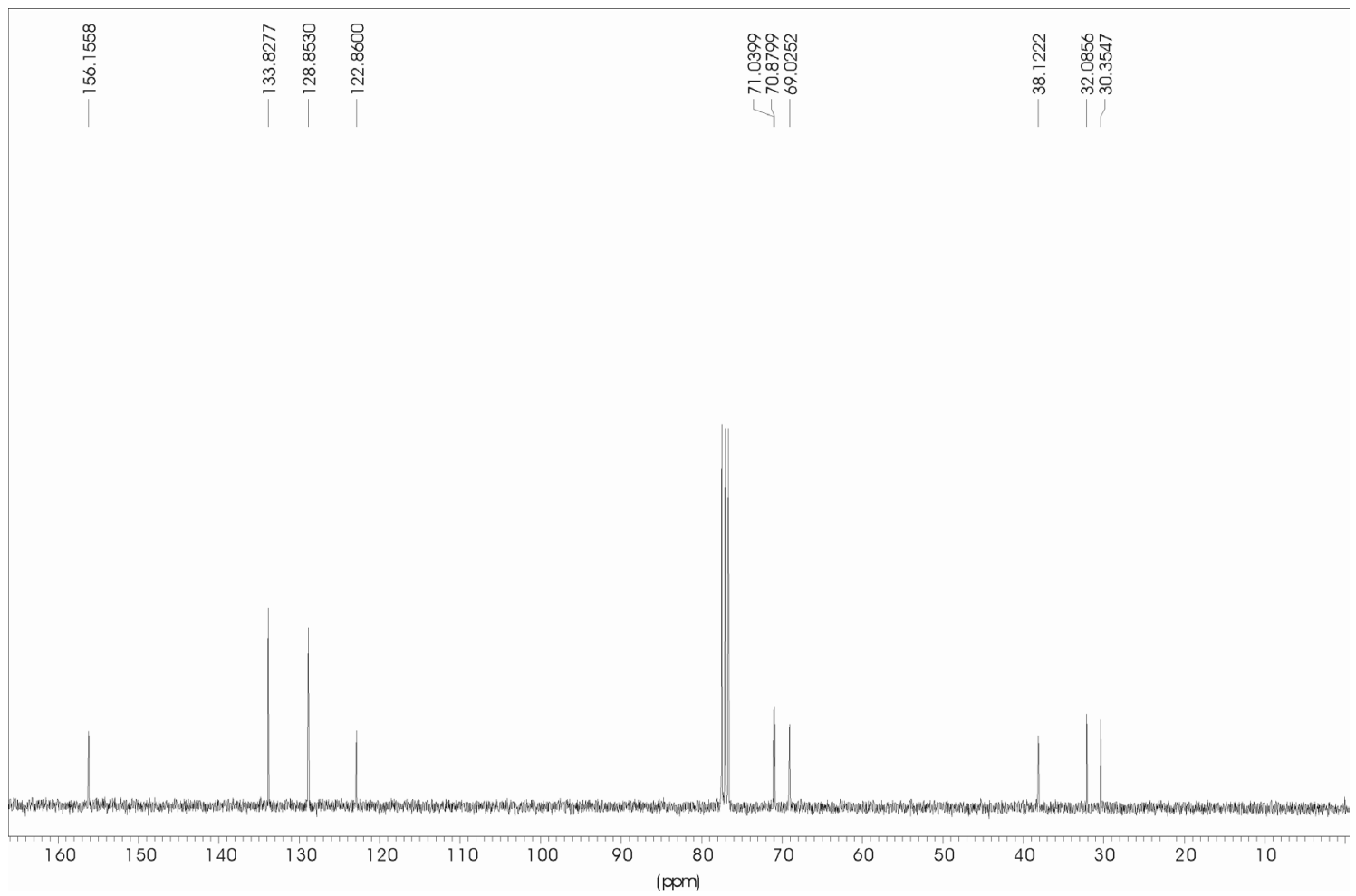

Figure $\mathbf{S 4} .{ }^{13} \mathrm{C}$ NMR spectrum of $\mathbf{L}^{\mathbf{2}}$. 


\section{Crystallographic Structure Determinations}

All data were collected on a Bruker Smart diffractometer equipped with a graphite monochromated Mo $K \alpha(\lambda=0.71073 \AA)$ radiation source and a CCD detector. The 45 frames of two dimensional diffraction images were collected and processed to obtain the cell parameters and orientation matrix. The first 50 frames were retaken after complete data collection. The crystal showed no significant decay. The frame data were processed to give structure factors using the SAINT. ${ }^{\mathrm{S} 1}$ The structure was solved by direct methods and refined by full matrix least squares methods on $F^{2}$ for all data using SHELXTL software. ${ }^{\text {S2 }}$ The nonhydrogen atoms were refined anisotropically. The atoms S2, C36, C42, C44, C45 and C46 in

$\mathbf{L}^{2}$ show relatively large thermal displacement parameters; attempts to resolve the atoms as partial occupancy could not be done sensibly. Crystal of $\mathbf{L}^{2}$ is characterized by noncentrosymmetric space symmetry, but was refined as racemic twins. The hydrogen atoms were placed in calculated positions and refined with a riding model with $U_{\text {iso }}$ constrained to be 1.2 times $U_{\text {eq }}$ of the parent atom except two disordered solvent molecules in $\mathbf{L}^{1}$. Relevant crystal data collection and refinement data for the crystal structures of $\mathbf{L}^{\mathbf{1}}, \mathbf{L}^{\mathbf{2}}$ and $\mathbf{1}$ are summarized in Table S1.

\section{References}

S1. Bruker, SMART and SAINT: Area Detector Control and Integration Software Ver. 5.0; Bruker Analytical X-ray Instruments: Madison, Wisconsin, 1998.

S2. Bruker, SHELXTL: Structure Determination Programs Ver. 5.16; Bruker Analytical X-ray Instruments: Madison, Wisconsin, 1998. 
Table S1. Crystal and experimental data

\begin{tabular}{|c|c|c|c|}
\hline & $\mathbf{L}^{1}$ & $\mathbf{L}^{2}$ & 1 \\
\hline Formula & $\mathrm{C}_{48.50} \mathrm{H}_{60} \mathrm{O}_{10} \mathrm{~S}_{2}$ & $\mathrm{C}_{48} \mathrm{H}_{60} \mathrm{O}_{8} \mathrm{~S}_{4}$ & $\mathrm{C}_{27} \mathrm{H}_{37} \mathrm{AgF}_{6} \mathrm{NO}_{5} \mathrm{PS}_{2}$ \\
\hline Formula weight & 867.08 & 893.20 & 772.54 \\
\hline Temperature (K) & $173(2)$ & $298(2)$ & $173(2)$ \\
\hline Crystal system & Monoclinic & Orthorhombic & Monoclinic \\
\hline Space group & $P 2_{1} / n$ & Pca2 ${ }_{1}$ & $C 2 / c$ \\
\hline$Z$ & 4 & 4 & 8 \\
\hline$a(\AA)$ & $14.0769(8)$ & $18.5095(16)$ & $16.4451(10)$ \\
\hline$b(\AA)$ & $10.8418(6)$ & $11.1086(10)$ & $17.1416(10)$ \\
\hline$c(\AA)$ & $30.9970(17)$ & $22.850(2)$ & $21.7675(12)$ \\
\hline$\alpha\left(^{\circ}\right)$ & 90 & 90 & 90 \\
\hline$\beta\left(^{\circ}\right)$ & $98.3330(10)$ & 90 & $96.0260(10)$ \\
\hline$\gamma\left({ }^{\circ}\right)$ & 90 & 90 & 90 \\
\hline$V\left(\AA^{3}\right)$ & $4680.8(5)$ & $4698.2(7)$ & $6102.3(6)$ \\
\hline$D_{x}\left(\mathrm{~g} / \mathrm{cm}^{3}\right)$ & 1.230 & 1.263 & 1.682 \\
\hline $2 \theta_{\max }$ & 56.58 & 56.64 & 56.54 \\
\hline$R$ & 0.0750 & 0.0808 & 0.0351 \\
\hline$w R$ & 0.2282 & 0.1449 & 0.0821 \\
\hline GOF & 1.093 & 1.092 & 1.053 \\
\hline \multicolumn{2}{|c|}{ Data / restraints / parameters 10899 / 0 / 597} & 10584 / 1 / 542 & $7168 / 0 / 389$ \\
\hline Absorption Correction & SADABS & SADABS & None \\
\hline \multirow[t]{2}{*}{ Diffractometer } & Bruker SMART & Bruker SMART & Bruker SMART \\
\hline & CCD system & CCD system & CCD system \\
\hline Structure determination & SHELXTL & SHELXTL & SHELXTL \\
\hline Refinement & full-matrix & full-matrix & full-matrix \\
\hline
\end{tabular}


Table S2. Selected bond lengths $(\AA)$, bond angles $\left({ }^{\circ}\right)$ and torsion angles $\left({ }^{\circ}\right)$ for $\mathbf{L}^{1}$

\begin{tabular}{|c|c|c|c|}
\hline $\mathrm{S}(1)-\mathrm{C}(38)$ & $1.804(3)$ & $\mathrm{S}(1)-\mathrm{C}(39)$ & $1.817(4)$ \\
\hline$S(2)-C(45)$ & $1.672(6)$ & $S(2)-C(44)$ & $1.711(14)$ \\
\hline$S\left(2^{\prime}\right)-C\left(44^{\prime}\right)$ & $1.802(10)$ & $S\left(2^{\prime}\right)-C(45)$ & $2.032(7)$ \\
\hline $\mathrm{O}(1)-\mathrm{C}(21)$ & $1.391(4)$ & $\mathrm{O}(1)-\mathrm{C}(37)$ & $1.437(4)$ \\
\hline $\mathrm{O}(9)-\mathrm{C}(1)$ & $1.417(4)$ & $\mathrm{O}(2)-\mathrm{C}(40)$ & $1.410(5)$ \\
\hline $\mathrm{O}(2)-\mathrm{C}(41)$ & $1.441(6)$ & $\mathrm{O}(8)-\mathrm{C}(2)$ & $1.423(4)$ \\
\hline $\mathrm{O}(3)-\mathrm{C}(43)$ & $1.329(17)$ & $\mathrm{O}(3)-\mathrm{C}(42)$ & $1.490(9)$ \\
\hline $\mathrm{O}\left(3^{\prime}\right)-\mathrm{C}(42)$ & $1.351(9)$ & $\mathrm{O}\left(3^{\prime}\right)-\mathrm{C}\left(43^{\prime}\right)$ & $1.37(3)$ \\
\hline $\mathrm{O}(8)-\mathrm{C}(3)$ & $1.417(5)$ & $\mathrm{O}(4)-\mathrm{C}(35)$ & $1.376(4)$ \\
\hline $\mathrm{O}(4)-\mathrm{C}(46)$ & $1.423(5)$ & $\mathrm{O}(7)-\mathrm{C}(4)$ & $1.420(5)$ \\
\hline $\mathrm{O}(5)-\mathrm{C}(9)$ & $1.390(4)$ & $\mathrm{O}(5)-\mathrm{C}(8)$ & $1.427(4)$ \\
\hline $\mathrm{O}(7)-\mathrm{C}(5)$ & $1.409(5)$ & $\mathrm{O}(6)-\mathrm{C}(6)$ & $1.418(5)$ \\
\hline $\mathrm{O}(6)-\mathrm{C}(7)$ & $1.424(4)$ & $\mathrm{O}(9)-\mathrm{C}(24)$ & $1.384(4)$ \\
\hline S1---S2 & $5.564(6)$ & O1---O4 & $3.825(3)$ \\
\hline O6---O8 & $5.600(4)$ & O5---O9 & $3.865(3)$ \\
\hline O2---O3 & $3.176(8)$ & & \\
\hline $\mathrm{C}(38)-\mathrm{S}(1)-\mathrm{C}(39)$ & $101.93(18)$ & $\mathrm{C}(45)-\mathrm{S}(2)-\mathrm{C}(44)$ & $100.5(5)$ \\
\hline $\mathrm{C}(21)-\mathrm{O}(1)-\mathrm{C}(37)$ & $113.9(2)$ & $\mathrm{C}\left(44^{\prime}\right)-\mathrm{S}\left(2^{\prime}\right)-\mathrm{C}(45)$ & $98.3(4)$ \\
\hline $\mathrm{C}(40)-\mathrm{O}(2)-\mathrm{C}(41)$ & $112.9(3)$ & $\mathrm{C}(43)-\mathrm{O}(3)-\mathrm{C}(42)$ & 114.0(9) \\
\hline $\mathrm{C}(42)-\mathrm{O}\left(3^{\prime}\right)-\mathrm{C}\left(43^{\prime}\right)$ & $112.9(17)$ & $\mathrm{C}(35)-\mathrm{O}(4)-\mathrm{C}(46)$ & $116.5(3)$ \\
\hline $\mathrm{C}(9)-\mathrm{O}(5)-\mathrm{C}(8)$ & $116.7(2)$ & $\mathrm{C}(6)-\mathrm{O}(6)-\mathrm{C}(7)$ & $114.5(3)$ \\
\hline $\mathrm{C}(5)-\mathrm{O}(7)-\mathrm{C}(4)$ & $112.2(3)$ & $\mathrm{C}(3)-\mathrm{O}(8)-\mathrm{C}(2)$ & $115.3(3)$ \\
\hline $\mathrm{C}(24)-\mathrm{O}(9)-\mathrm{C}(1)$ & $115.0(2)$ & & \\
\hline $\mathrm{S}(1)-\mathrm{C}(38)-\mathrm{C}(37)-\mathrm{O}(1)$ & $-177.3(3)$ & $\mathrm{S}(1)-\mathrm{C}(39)-\mathrm{C}(40)-\mathrm{O}(2)$ & $-69.2(4)$ \\
\hline $\mathrm{S}(2)-\mathrm{C}(45)-\mathrm{C}(46)-\mathrm{O}(4)$ & $-161.5(4)$ & $\mathrm{S}(2)-\mathrm{C}(44)-\mathrm{C}(43)-\mathrm{O}(3)$ & $-176.3(10)$ \\
\hline $\mathrm{O}(3)-\mathrm{C}(42)-\mathrm{C}(41)-\mathrm{O}(2)$ & $-85.8(7)$ & $\mathrm{O}(6)-\mathrm{C}(7)-\mathrm{C}(8)-\mathrm{O}(5)$ & $-166.6(3)$ \\
\hline $\mathrm{O}(7)-\mathrm{C}(5)-\mathrm{C}(6)-\mathrm{O}(6)$ & $78.5(5)$ & $\mathrm{O}(8)-\mathrm{C}(3)-\mathrm{C}(4)-\mathrm{O}(7)$ & $-79.8(5)$ \\
\hline $\mathrm{O}(9)-\mathrm{C}(1)-\mathrm{C}(2)-\mathrm{O}(8)$ & $-177.3(3)$ & & \\
\hline \multicolumn{4}{|l|}{ Dihedral Angles } \\
\hline A---B & $78.57(11)$ & A---C & $47.10(12)$ \\
\hline A---D & $79.57(9)$ & B---C & $82.40(9)$ \\
\hline B---D & $42.72(14)$ & C---D & $86.32(9)$ \\
\hline \multicolumn{4}{|c|}{ Centroid-centroid distances } \\
\hline A---C & 6.145 & B---D & 6.057 \\
\hline
\end{tabular}


Table S3. Selected bond lengths $(\AA)$, bond angles $\left(^{\circ}\right)$ and torsion angles $\left({ }^{\circ}\right)$ for $\mathbf{L}^{2}$

\begin{tabular}{|c|c|c|c|}
\hline$S(1)-C(31)$ & $1.800(6)$ & $S(1)-C(30)$ & $\overline{1.815(6)}$ \\
\hline$S(2)-C(36)$ & $1.748(10)$ & $S(2)-C(37)$ & $1.772(6)$ \\
\hline$S(3)-C(41)$ & $1.803(7)$ & $S(3)-C(40)$ & $1.811(6)$ \\
\hline$S(4)-C(46)$ & $1.773(8)$ & $S(4)-C(47)$ & $1.810(6)$ \\
\hline $\mathrm{O}(1)-\mathrm{C}(6)$ & $1.384(6)$ & $\mathrm{O}(1)-\mathrm{C}(29)$ & $1.423(6)$ \\
\hline $\mathrm{O}(2)-\mathrm{C}(32)$ & $1.427(7)$ & $\mathrm{O}(2)-\mathrm{C}(33)$ & $1.432(8)$ \\
\hline $\mathrm{O}(3)-\mathrm{C}(34)$ & $1.397(8)$ & $\mathrm{O}(3)-\mathrm{C}(35)$ & $1.429(9)$ \\
\hline $\mathrm{O}(4)-\mathrm{C}(20)$ & $1.383(6)$ & $\mathrm{O}(4)-\mathrm{C}(38)$ & $1.412(6)$ \\
\hline $\mathrm{O}(5)-\mathrm{C}(13)$ & $1.380(5)$ & $\mathrm{O}(5)-\mathrm{C}(39)$ & $1.406(6)$ \\
\hline $\mathrm{O}(6)-\mathrm{C}(42)$ & $1.414(9)$ & $\mathrm{O}(6)-\mathrm{C}(43)$ & 1.433(9) \\
\hline $\mathrm{O}(7)-\mathrm{C}(45)$ & $1.204(9)$ & $\mathrm{O}(7)-\mathrm{C}(44)$ & $1.548(12)$ \\
\hline $\mathrm{O}(8)-\mathrm{C}(27)$ & $1.394(6)$ & $\mathrm{O}(8)-\mathrm{C}(48)$ & $1.424(6)$ \\
\hline $\mathrm{C}(31)-\mathrm{S}(1)-\mathrm{C}(30)$ & $101.5(3)$ & $C(36)-S(2)-C(37)$ & $102.6(3)$ \\
\hline $\mathrm{C}(41)-\mathrm{S}(3)-\mathrm{C}(40)$ & $100.0(3)$ & $\mathrm{C}(46)-\mathrm{S}(4)-\mathrm{C}(47)$ & 101.3(3) \\
\hline $\mathrm{C}(6)-\mathrm{O}(1)-\mathrm{C}(29)$ & $117.7(4)$ & $\mathrm{C}(32)-\mathrm{O}(2)-\mathrm{C}(33)$ & $115.1(5)$ \\
\hline $\mathrm{C}(34)-\mathrm{O}(3)-\mathrm{C}(35)$ & $112.4(7)$ & $\mathrm{C}(20)-\mathrm{O}(4)-\mathrm{C}(38)$ & $116.7(4)$ \\
\hline $\mathrm{C}(13)-\mathrm{O}(5)-\mathrm{C}(39)$ & $117.5(4)$ & $\mathrm{C}(42)-\mathrm{O}(6)-\mathrm{C}(43)$ & $112.5(7)$ \\
\hline $\mathrm{C}(45)-\mathrm{O}(7)-\mathrm{C}(44)$ & $95.8(10)$ & $\mathrm{C}(27)-\mathrm{O}(8)-\mathrm{C}(48)$ & $112.5(4)$ \\
\hline $\mathrm{S}(1)-\mathrm{C}(30)-\mathrm{C}(29)-\mathrm{O}(1)$ & $-175.6(4)$ & $\mathrm{S}(1)-\mathrm{C}(31)-\mathrm{C}(32)-\mathrm{O}(2)$ & 179.7(4) \\
\hline $\mathrm{S}(2)-\mathrm{C}(37)-\mathrm{C}(38)-\mathrm{O}(4)$ & $-163.3(4)$ & $\mathrm{S}(2)-\mathrm{C}(36)-\mathrm{C}(35)-\mathrm{O}(3)$ & $-53.3(8)$ \\
\hline $\mathrm{S}(3)-\mathrm{C}(40)-\mathrm{C}(39)-\mathrm{O}(5)$ & $180.0(3)$ & $\mathrm{S}(3)-\mathrm{C}(41)-\mathrm{C}(42)-\mathrm{O}(6)$ & $146.7(4)$ \\
\hline $\mathrm{S}(4)-\mathrm{C}(46)-\mathrm{C}(45)-\mathrm{O}(7)$ & $-67.4(10)$ & $\mathrm{S}(4)-\mathrm{C}(47)-\mathrm{C}(48)-\mathrm{O}(8)$ & $-172.1(4)$ \\
\hline $\mathrm{O}(2)-\mathrm{C}(33)-\mathrm{C}(34)-\mathrm{O}(3)$ & $78.4(8)$ & $\mathrm{O}(6)-\mathrm{C}(43)-\mathrm{C}(44)-\mathrm{O}(7)$ & $-55.2(13)$ \\
\hline$S(1)---S(2)$ & $5.631(4)$ & $S(3)---S(4)$ & $6.326(3)$ \\
\hline \multicolumn{2}{|c|}{$\mathrm{O}(1)$--- $\mathrm{O}(4)$} & $\mathrm{O}(2)--\mathrm{O}(3)$ & \\
\hline $\mathrm{O}(5)$--- $\mathrm{O}(8)$ & $3.908(5)$ & $O(6)---O(7)$ & $3.051(10)$ \\
\hline
\end{tabular}

Dihedral Angles

$\begin{array}{llll}\text { A---B } & 80.50(13) & \text { A---C } & 42.83(19) \\ \text { A---D } & 82.40(14) & \text { B---C } & 86.09(16) \\ \text { B---D } & 44.35(20) & \text { C---D } & 79.38(12)\end{array}$

Centroid-centroid distances

A---C

6.071

B---D

6.064 
Table S4. Selected bond lengths $(\AA)$, bond angles $\left(^{\circ}\right)$ and torsion angles $\left(^{\circ}\right)$ for $\underline{\left[\mathrm{Ag}_{2}\left(\mathbf{L}^{\mathbf{2}}\right)\right]\left(\mathrm{PF}_{6}\right)_{2}(\mathrm{DMF})_{2}, \mathbf{1}}$

\begin{tabular}{|c|c|c|c|}
\hline $\operatorname{Ag}(1)-S(1)$ & $2.481(1)$ & $\operatorname{Ag}(1)-S(2)$ & $2.510(1)$ \\
\hline$S(1)-C(17)$ & $1.818(2)$ & $S(1)-C(18)$ & $1.820(3)$ \\
\hline$S(2)-C(24)$ & $1.816(2)$ & $S(2)-C(23)$ & $1.817(3)$ \\
\hline $\mathrm{O}(1)-\mathrm{C}(7)$ & $1.391(3)$ & $\mathrm{O}(1)-\mathrm{C}(16)$ & $1.439(3)$ \\
\hline $\mathrm{O}(2)-\mathrm{C}(19)$ & $1.426(3)$ & $\mathrm{O}(2)-\mathrm{C}(20)$ & $1.427(3)$ \\
\hline $\mathrm{O}(3)-\mathrm{C}(21)$ & $1.408(3)$ & $\mathrm{O}(3)-\mathrm{C}(22)$ & $1.422(3)$ \\
\hline $\mathrm{O}(4)-\mathrm{C}(14 \mathrm{~A})$ & $1.388(3)$ & & \\
\hline $\mathrm{Ag}(1)---\mathrm{O}(1)$ & $3.754(2)$ & $\mathrm{Ag}(1)---\mathrm{O}(2)$ & $3.115(2)$ \\
\hline $\mathrm{Ag}(1)---\mathrm{O}(3)$ & $2.710(2)$ & $\mathrm{Ag}(1)---\mathrm{O}(4)$ & $3.728(2)$ \\
\hline $\operatorname{Ag}(1)---C(11)$ & $3.642(3)$ & $\operatorname{Ag}(1)---C(4 A)$ & $2.818(2)$ \\
\hline$S(1)---S(2)$ & 4.631(1) & $\mathrm{O}(1)---\mathrm{O}(4)$ & $4.986(2)$ \\
\hline $\mathrm{O}(2)---\mathrm{O}(3)$ & $2.787(3)$ & $\operatorname{Ag}(1)---\operatorname{Ag}(1 \mathrm{~A})$ & $8.163(1)$ \\
\hline$S(1)-\operatorname{Ag}(1)-S(2)$ & $136.24(2)$ & $\mathrm{C}(17)-\mathrm{S}(1)-\mathrm{C}(18)$ & $98.66(12)$ \\
\hline$C(17)-S(1)-\operatorname{Ag}(1)$ & $118.20(8)$ & $C(18)-S(1)-A g(1)$ & $104.20(9)$ \\
\hline$C(24)-S(2)-C(23)$ & $98.14(12)$ & $C(24)-S(2)-\operatorname{Ag}(1)$ & $112.42(9)$ \\
\hline$C(23)-S(2)-\operatorname{Ag}(1)$ & $102.61(10)$ & $\mathrm{C}(7)-\mathrm{O}(1)-\mathrm{C}(16)$ & $113.63(17)$ \\
\hline $\mathrm{C}(19)-\mathrm{O}(2)-\mathrm{C}(20)$ & $112.7(2)$ & $\mathrm{C}(21)-\mathrm{O}(3)-\mathrm{C}(22)$ & $113.9(2)$ \\
\hline $\mathrm{C}(14 \mathrm{~A})-\mathrm{O}(4)-\mathrm{C}(25)$ & $112.80(17)$ & & \\
\hline $\mathrm{O}(1)-\mathrm{C}(16)-\mathrm{C}(17)-\mathrm{S}(1)$ & $61.7(2)$ & $\mathrm{S}(1)-\mathrm{C}(18)-\mathrm{C}(19)-\mathrm{O}(2)$ & $63.6(3)$ \\
\hline $\mathrm{O}(2)-\mathrm{C}(20)-\mathrm{C}(21)-\mathrm{O}(3)$ & $62.8(3)$ & $\mathrm{O}(3)-\mathrm{C}(22)-\mathrm{C}(23)-\mathrm{S}(2)$ & $-66.3(3)$ \\
\hline $\mathrm{S}(2)-\mathrm{C}(24)-\mathrm{C}(25)-\mathrm{O}(4)$ & $-60.7(4)$ & & \\
\hline $\operatorname{Ag}(1)---D$ centroid & 3.495 & Ag(1)--- B centroid & 4.198 \\
\hline \multicolumn{4}{|l|}{ Dihedral Angles } \\
\hline A---B & $88.79(6)$ & B---D & $14.47(13)$ \\
\hline A---D & $87.97(6)$ & & \\
\hline \multicolumn{4}{|c|}{ Centroid-centroid distance } \\
\hline A---C & 5.569 & & \\
\hline
\end{tabular}

[Symmetry codes A: $-x, y,-z+3 / 2]$ 
Table S5. Comparison of the structural parameter of the calix[4] unit for free $\mathbf{L}^{2}$ and $\mathbf{1}$.

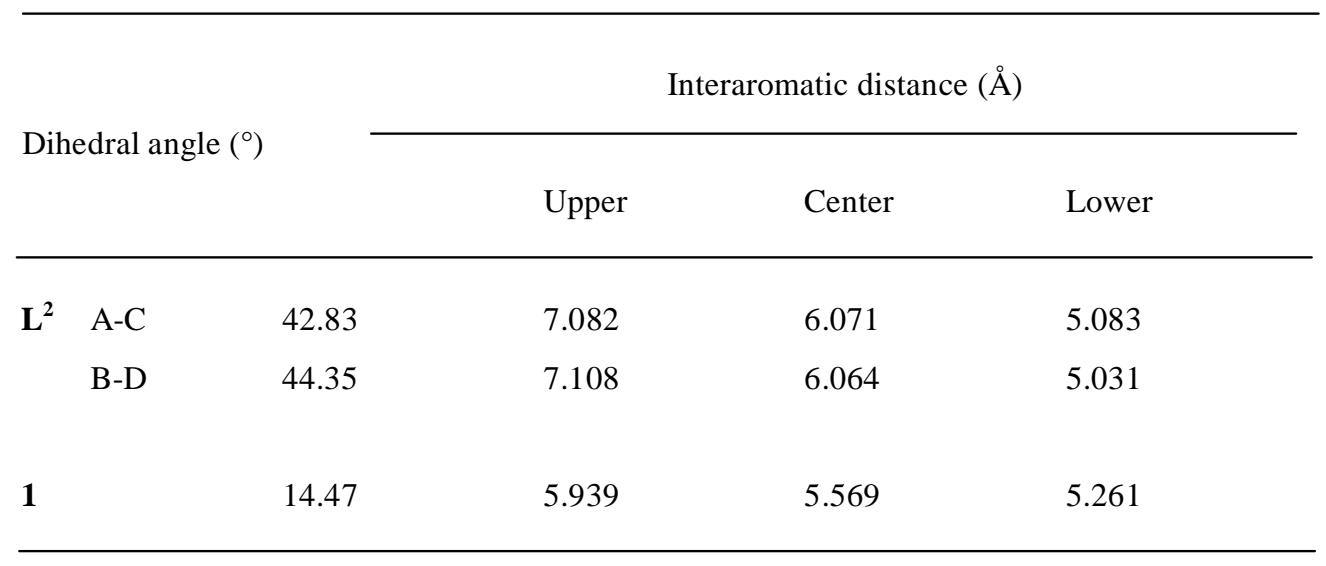




\section{Solvent Extraction}

The evaluation of the binding efficiencies of the calix[4]thiacrowns $\mathbf{L}^{1}-\mathbf{L}^{2}$ were carried out by two-phase solvent extraction of metal picrates $\left(\mathrm{Ag}^{+}, \mathrm{Na}^{+}, \mathrm{K}^{+} \mathrm{Rb}^{+}, \mathrm{Cs}^{+}, \mathrm{NH}_{4}^{+}, \mathrm{Pb}^{2+}, \mathrm{Sr}^{2+}\right.$, and $\mathrm{Ba}^{2+}$ ) into 1,2-dichloroethane under neutral conditions. The extracted amount of each metal ion by the calix[4]thiacrown extractant was measured conveniently by the determination of picrate ion which extracted as a complexed ion-pair form, $\mathbf{M}^{\mathrm{n}+} \cdot \mathbf{L} \cdot\left(\mathrm{pic}^{-}\right)_{\mathrm{n}}$. The results are shown in Figure 4 (see also Table S6). Since the ionophores employed have two possible binding sites for the metal species and in order to enable direct comparison with prior extraction results for the related systems employing calix[4]-bis-crowns, the concentrations of ionophores in organic phase $(2.00 \mathrm{~mL})$ and metal picrate in aqueous phase $(2.00 \mathrm{~mL})$ also set at $1.00 \times 10^{-4} \mathrm{M}$ and $2.00 \times 10^{-4} \mathrm{M}$, respectively. Thus the maximum extractability can be defined as $200 \%$ :

$$
\text { Extractability }=\left\{[\text { Extracted } \text { metal picrate }]_{\text {org }} /[\text { [onophore }]_{\text {org }}\right\} \times 100(\%)
$$

A water-saturated 1,2-dichloroethane solution of macrocycle $\left(2.00 \mathrm{~mL}, 1.00 \times 10^{-4} \mathrm{M}\right)$ and an aqueous solution of metal picrate $\left(2.00 \mathrm{~mL}, 2.00 \times 10^{-4} \mathrm{M}\right)$ were placed in a volumetruic flask, and the flask was shaken in a thermostated water bath for $1 \mathrm{~h}$ at $25 \pm 0.2{ }^{\circ} \mathrm{C}$. After standing for $2 \mathrm{~h}$, the concentration of metal picrate in the aqueous phase was determined at $356 \mathrm{~nm}$ with Scinco S-3100 UV/Vis spectrophotometer.

Table S6. Extractability of metal picrates from aqueous to 1,2-dichloroethane at $25^{\circ} \mathrm{C}$

\begin{tabular}{lcclllllll} 
& $\mathrm{Na}^{+}$ & $\mathrm{K}^{+}$ & $\mathrm{Rb}^{+}$ & $\mathrm{Cs}^{+}$ & $\mathrm{NH}_{4}^{+}$ & $\mathrm{Ag}^{+}$ & $\mathrm{Pb}^{2+}$ & $\mathrm{Sr}^{2+}$ & $\mathrm{Ba}^{2+}$ \\
\hline $\mathbf{L}^{\mathbf{1}}$ & 47 & 122 & 120 & 112 & 120 & 189 & 62 & 45 & 49 \\
$\mathbf{L}^{2}$ & $\mathbf{7}$ & 5 & 12 & 19 & 7 & 194 & 6 & 10 & 10 \\
\hline
\end{tabular}




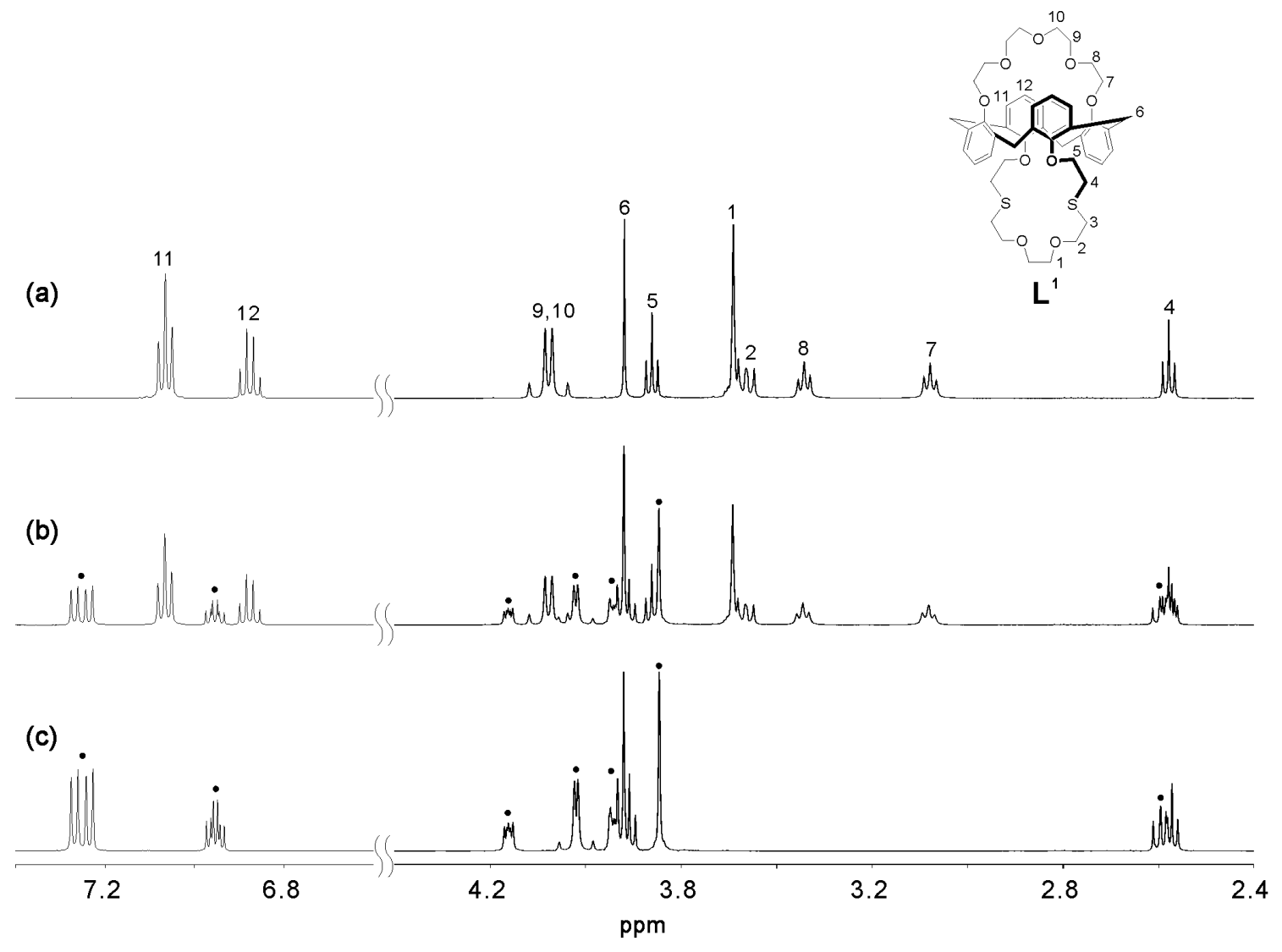

Figure S5. Partial ${ }^{1} \mathrm{H}-\mathrm{NMR}\left(500 \mathrm{MHz}\right.$ ) spectra for (a) free $\mathbf{L}^{\mathbf{1}}(0.1 \mathrm{mM})$, (b) $\mathbf{L}^{\mathbf{1}}+0.4$ equiv KPic, and (c) $\mathbf{L}^{\mathbf{1}}+$ 1.0 equiv KPic in $\mathrm{CD}_{3} \mathrm{CN} / \mathrm{CDCl}_{3}(v / v 1: 1)$. Peaks with dot refer to complexed $\mathbf{L}^{1}$. 

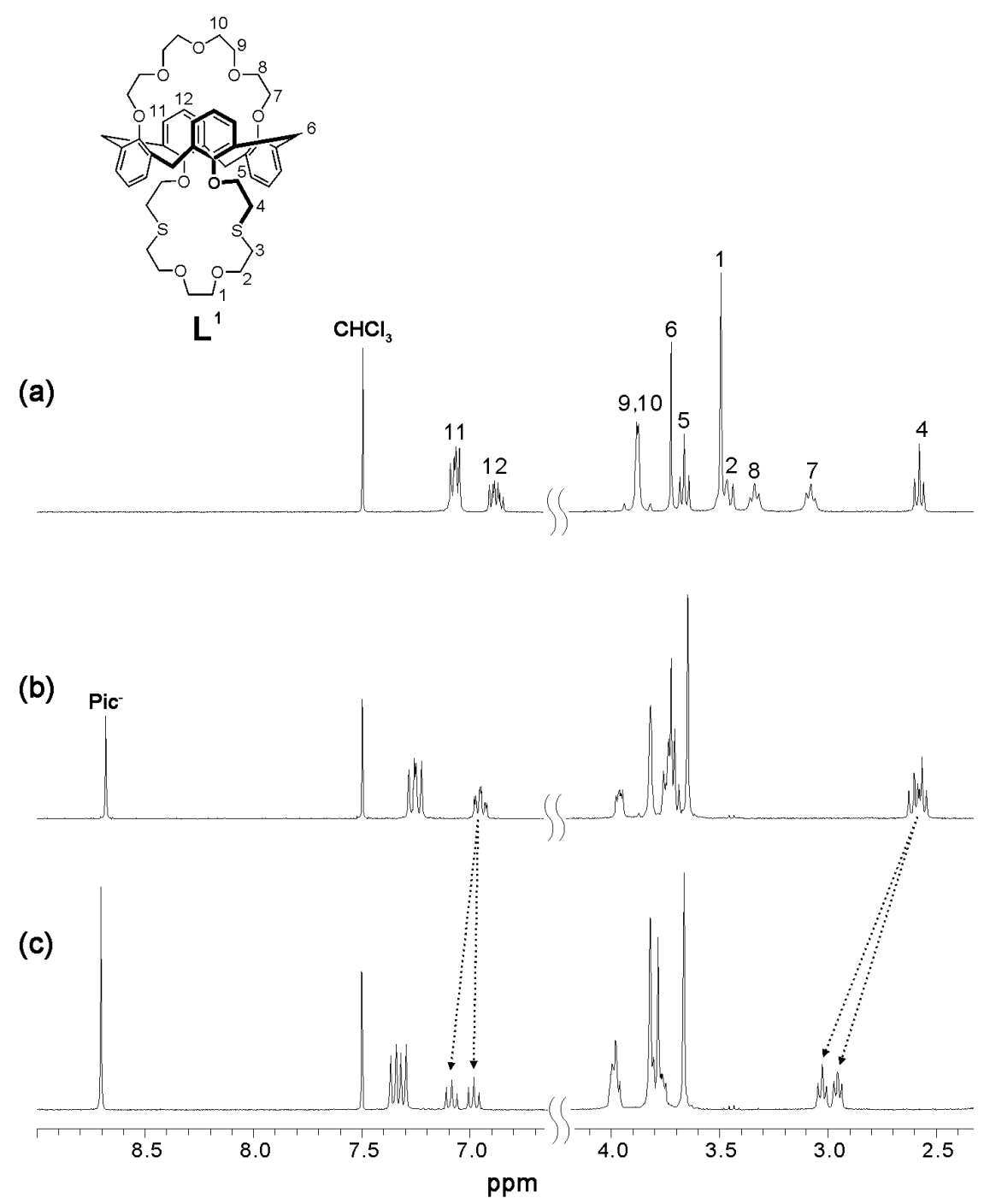

Figure S6. Partial ${ }^{1} \mathrm{H}-\mathrm{NMR}(300 \mathrm{MHz})$ spectra for (a) free $\mathbf{L}^{\mathbf{1}}(0.1 \mathrm{mM})$, (b) $\mathbf{L}^{\mathbf{1}}+1.0$ equiv KPic, and (c) $\mathbf{L}^{\mathbf{1}}+$ 1.0 equiv KPic +1.0 equiv $\mathrm{AgPic}$ in $\mathrm{CD}_{3} \mathrm{CN} / \mathrm{CDCl}_{3}(v / v 1: 1)$. 


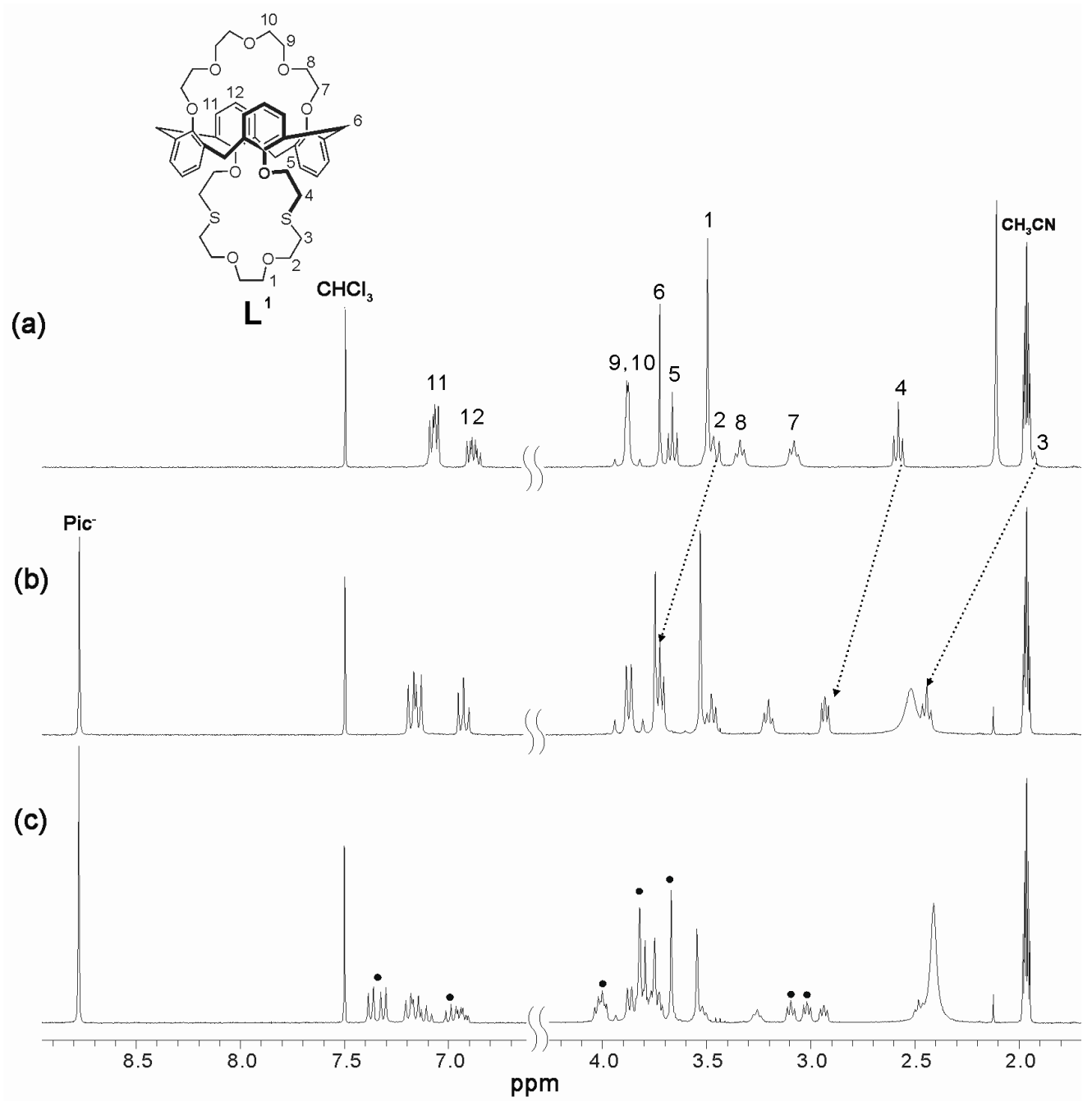

Figure S7. Partial ${ }^{1} \mathrm{H}-\mathrm{NMR}(300 \mathrm{MHz})$ spectra for (a) free $\mathbf{L}^{\mathbf{1}}(0.1 \mathrm{mM})$, (b) $\mathbf{L}^{\mathbf{1}}+2.0$ equiv AgPic, and (c) $\mathbf{L}^{\mathbf{1}}+$ 2.0 equiv $\mathrm{AgPic}+1.0$ equiv $\mathrm{KPic}$ in $\mathrm{CD}_{3} \mathrm{CN} / \mathrm{CDCl}_{3}(v / v 1: 1)$. 


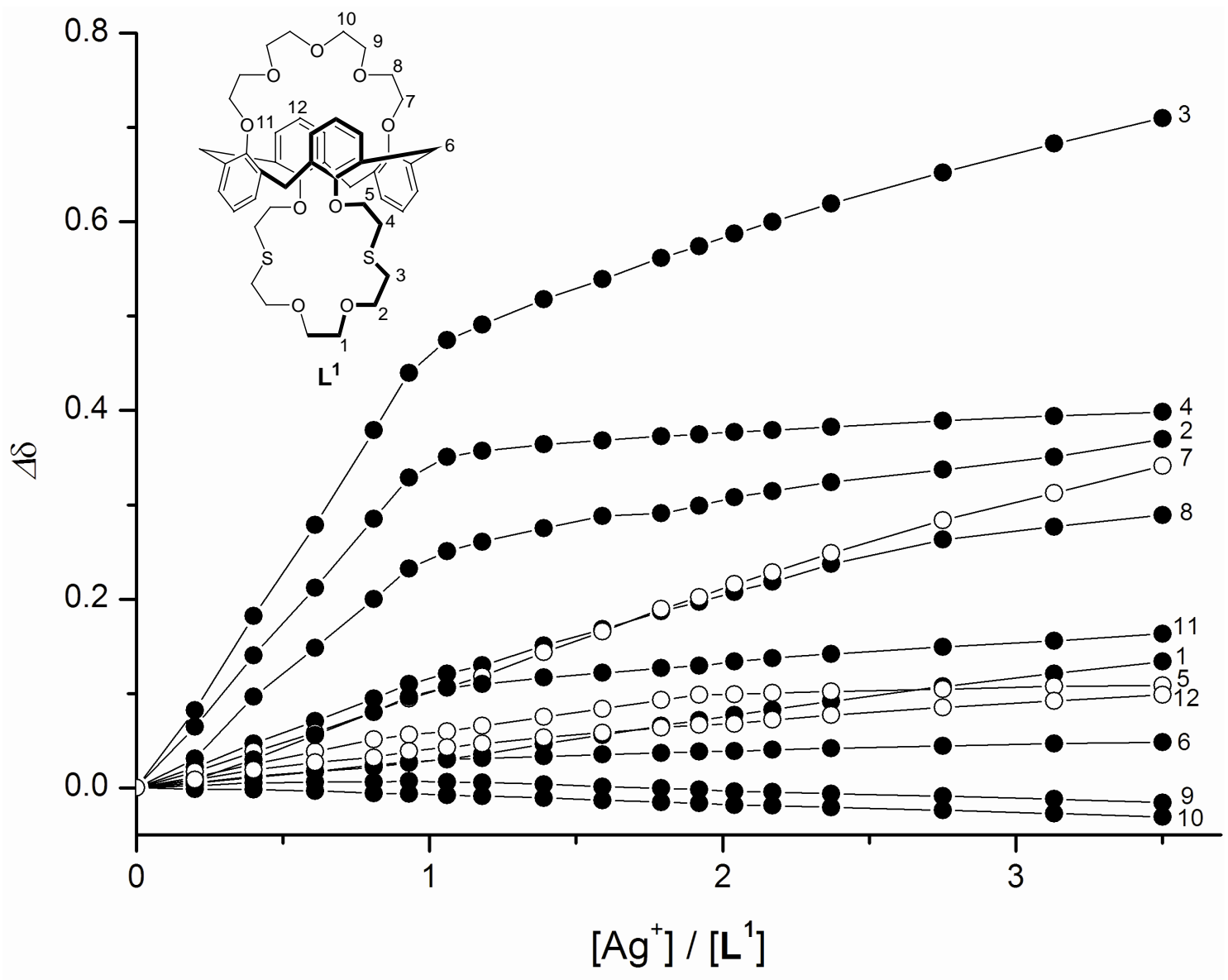

Figure S8. NMR titration plots of $\mathbf{L}^{\mathbf{1}}$ with silver perchlorate in $\mathrm{CD}_{3} \mathrm{CN}_{-} \mathrm{CDCl}_{3}(\mathrm{v} / \mathrm{v}$ 1:1). 


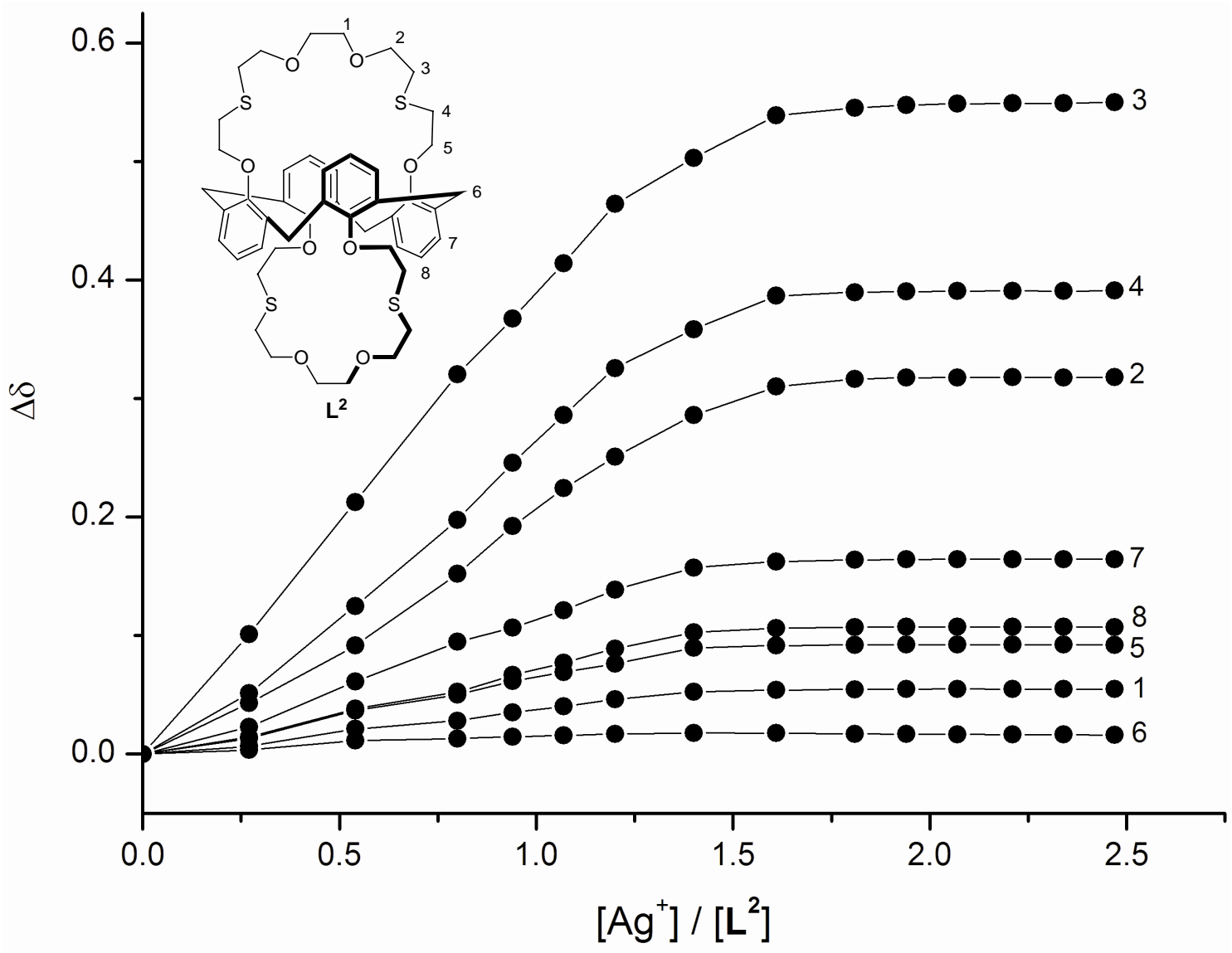

Figure S9. NMR titration plots of $\mathbf{L}^{2}$ with silver per chlorate in $\mathrm{CD}_{3} \mathrm{CN}-\mathrm{CDCl}_{3}(\mathrm{v} / \mathrm{v}$ 1:1). 